\section{Aplicación práctica de la Norma de Información Financiera}

\section{C1 -Efectivo.}

Practical application of Mexican Financial Reporting Standard C 1 -Cash.

\section{Ana Elsa Ortiz Noriega}

Sergio Félix Enríquez ${ }^{2}$

\section{Resumen}

El rubro de efectivo, es considerado como el más importante de toda entidad económica. Es el que da liquidez a la La NIF C1 tiene varios años de emitida, en el 2001 se modimpresa, comprar, vender, invertir, gastar, todo está refleja- fica el antiguo boletín C1, en 2008 se emite la NIF B-2 Estado en términos de dinero. Para cumplir cabalmente con el de Flujo de efectivo por parte del CINIF, estableciendo las bacontrol de efectivo se requiere seguir al pie de la letra la nor- ses para determinar el estado de flujo de efectivo haciendo

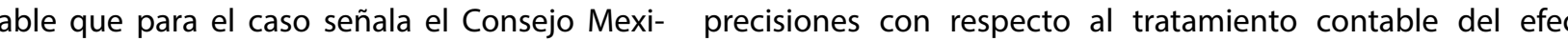
de Normas de Información Financiera (CINIF). El efec- tivo modificando el boletín C1, dando paso a la NIF C-1, la tivo está regulado por la Norma de Información Financiera cual se analiza en este artículo desde las disposiciones teóC1 (NIF C1) por lo que el presente trabajo pretende dar un ricas y mediante la resolución de algunos casos prácticos. panorama claro sobre la aplicación práctica de la NIF $\mathrm{C}$.

Palabras clave: Normas de Información Financiera, Efectivo,
valuación

\section{Abstract}

The caption cash, is considered the most important of all economic entity. It is what gives liquidity to the printed, buy, sell, invest, spend, everything is reflected in terms of money. To comply fully with effective control is required follow to the letter the accounting standards for the case marks the Mexican Board of $\mathrm{F}-$ nancial Reporting Standards (CINIF). Cash is regulated by the Fito give a clear picture on the practical application of MFRS C1.

\section{Keywords: Financial Reporting, Cash, valuation}

La NIF C1 (IMPC 2014), dice en sus normas de valuación, que e efectivo debe valuarse a suvalor nominal Todos los equivalentes de efectivo en su reconocimiento inicial deben reconocerse a su costo de adquisición. Dentro del reconocimiento posterior de los equivalentes de efectivo, representados por metales preciosos monedados, deben valuarse a su valor razonable; para tal efecto, debe utilizarse su valor neto de realización a la fecha de cierre $d$ . estado de resultados conforme se devenguen. Los equivalentes de efectivo denominados en monedas extranjeras deben convertirse o reexpresarse a la moneda de informe utilizado el tipo de cambio con el que pudieron haberse realizado a la fecha decierre de los esta dos financieros. los efectos de tales conversiones deben

El objetivo es establecer las normas que sirvan para valuar presentar y revelar las partidas que inegrana para valuar, presentar y revelar las partidas que integran el concepto de financier $y$ es aplicable a todas las entidades que emitan estados financieros en los términos de la NIF A-3 Necesida

Contador Público, Doctora en Administración Educativa Profesor investigador de tiempo completo de la Acade hia de Contabilidad Básica del Departamento de Contabilidad de la Universidad de Sonora, México. Email aeortį@itic.uson.mx Contador Público. Doctor Administración educativa. Profesor de tiempo completo de la Academia de Contabilidad Básica del Departa-

8 / Trascender, Contabilidad y Gestión

\title{
3. Valuación.
}

1. El efectivo debe valuarse a su valor nominal.

2. Los equivalentes de efectivo en su reconocimiento inicial deben reconocerse a su costo de adquisicion.

\section{Presentación.}

1) Este concepto debe presentarse en un solo renglón como e primer rubro del activo a corto plazo incluyendo el efectivo y equivalentes de efectivo restringido. El renglón debe llamarse: Efectivo y Equivalentes de Efectivo. Si la restricción expira en fecha posterior a los doce meses de la fecha del estado de situación financiera debe presentarse en el activo a largo plazo con e

\section{Definición de conceptos}

a) Costo de Adquisición. Es el monto pagado de efectivo o equivalente por un activo o servicio al momento de la adquisición.

b) Efectivo. Es la moneda de curso legal en caja y en depósitos bancarios disponibles para la operación de la entidad.

c) Equivalentes de Efectivo. Son valores de corto plazo, de gran quidez fácilmente convertible a efectivo y sujetos a riesgos poco mportantes de cambios en su valor.

d) Efectivo y equivalentes de efectivo, restringibles. Son as cuales normalmente son de tipo contractual o legal.

e) Inversiones disponibles a la vista. Son valores cuya disposición por parte de la entidad se prevé de forma inmediata, generan rendimientos y tienen riesgos poco importantes de cambio en su valor.

Valor neto de realización. es el monto que se recibe de efect, equivalente de efectivo o en especie por la venta o intercambio de un activo.

Valor nominal es la cantidad de unid das en billetes, monedas, títulos e instrumentos.

h) Valor razonable. Representa el monto de efectivo o equivalente de efectivo que participantes en el mercado estaría dispuestos a intercambiar para la compra y venta de un activo o para asumir o liquidar un pasivo, en una operación entre parcompetencia.

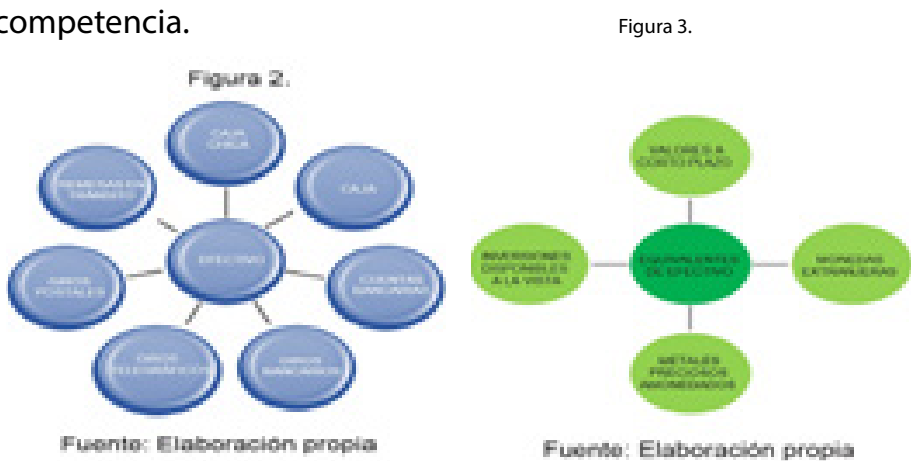

2) Los cheques emitidos que estén pendientes de entrega en la fecha de los estados financieros deben reincorporarse al

3) Cuando no se tengan convenios de compensación con la ins-

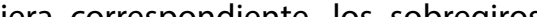
deben mostrarse como un pasivo a corto plazo, aun cuando se mantengan otras cuentas de cheques con saldo deudor en la misma institución

Los intereses devengados y las fluctuaciones cambiarias de los equivalentes de efectivo asi como los ajustes a valor razonable de los equivalentes de efectivo deben presentarse en el resultado integral de financiamiento según la NIF B-3.

\section{5evelación}

Las notas de los estados financieros deben revelar información con respecto a:

La integración del efectivo, equivalente de efectivo y política de valuación y moneda o medida de intercambio.

2) Si existe efectivo y equivalente de efectivo restringido, mencionar su importe y las causas.

3) Mencionar los importes de efectivo o equivalente de efectivo que estén destinados a un fin específico por parte de la administración.

Efectos de los hechos posteriores que por su importancia hayan modificado sustancialmente la valuación del efectivo en moneda extraniera metales preciosos amonedados y en inversiones disponibles a la vista.

Casos prácticos.

Para poner en práctica la aplicación de las normas de valuación presentación y revelación del Efectivo y equivalente de efectivo dispuesto en NIF C1, a continuación de presentan varios casos resueltos.

Núm. 1 / Enero- Abril del 2016 


\section{Apéndices Técnicos}

Caso 1

Caso 2.

Ferretera San Pedro, S.A. tiene en su cuenta de bancos un saldo Maderas Finas del Noroeste, S.A. tiene en su cuenta de Banco de $\$ 23,800.00$ en moneda nacional y en moneda extranjera un $\$ 123,500.00$ en moneda extranjera en caja 5,000.00 USD y cuensaldo de 1,250.00 USD, el tipo de cambio en su fecha de adqui- ta con dos centenarios depositados en caja de seguridad bancasición fue de 12.58 y en la fecha de la elaboración de los estados ria. Para efectos de presentación en el estado de situación confinancieros es de 12.90. En la cuenta de cheques en moneda na- sidera el t.c. vigente del dólar bancario a 13.05 y la cotización de cional se tienen cheques emitidos por un monto de $\$ 3,450.00$ los valores de metales preciosos amonedados es de $\$ 6,450.00$ los cuales no se han entregado a sus beneficiarios. efectivo es el siguiente:

Ferretería San Pedro

Estado de Situación Financiera al 31 de diciembre de 20.

Activo

Pasivo

Circulante

Capital Contable

Hermosillo, Sonora a 31 de diciembre de 20

Notas:

Integración de efectivo: Cuenta Bancaria No. 0001 Banamex S.A. Saldo $\$ 27,250,00$ 2.Equivalente en efectivo: Cuenta Bancaria Moneda Extranjer
Cta..... $\$ 16,125,00$ equivalente a 1250.00 USD al T.C. de 12.90

Nota al caso No. 1

De acuerdo con las normas de valuación el efectivo debe valuarse a su valor nominal, y equivalente de efectivo en este caso moneda extranjera a su costo de adquisición: 1250 x 12.58=15,725.00. La norma de presentación debe reconocer el cambio y valuarse al T.C. vigente en la fecha del estado financiero, reconociendo las fluctuaciones cambiarias como parte del resultado integral, en
esta situación la diferencia de $16,125.00-15,725.00=\$ 400.00$ y los cheques emitidos no entregados se reintegran al saldo bancienclo del concepto cubierto por dichos cheques.

De la misma manera se debe informar a través de las notas a los estados financieros, como se integra el rubro de efectivo, equi-

valente a efectivo, el tipo de cambio de conversión o reexpresión. sentar y revelar en los estados financieros es la siguiente:

Notas al caso 2

Maderas Finas del Noroeste, S.A.

Estado de Situación Financiera al 31 de diciembre de 20

Activo

Circulante

de Efectivo

Capital Contable

Hermosillo, Sonora a 31 de diciembre de 20.

Notas:

1. El rubro de efectivo se integra por: Bancos con saldo de $\$ 123,500.002$. El rubro de equivalente de efectivo se integra por:

$\begin{array}{ll}\text { Moneda Extranjera } & \$ 65,250.00 \\ \text { Metales preciosos amonedados } & \$ 12,900.00\end{array}$

2. El tipo de cambio utilizado para M.E. es de $13.05 \times 1$ y la cotización de metales preciosos amonedados es de $\$ 6,450.00 \times 1$

Supermercados al Menudeo, S.A. obtiene de su contabilidad la siguiente información de sus cuentas de efectivo y equivalentes para la preparación de la informacion financiera.

En las cuentas de Bancos moneda nacional tiene un saldo de $\$ 213,500.00$ en total, una de estas cuentas muestra un sobre giro de $\$ 3,520.00$. (No existiendo convenio con la institución financiera para este fin). De acuerdo con el contrato de servicios, el banco le exige que mantenga un saldo sin derecho a sutro por $\$ 20,000.00$ cada mes para cubiri contingencias con sus líneas de crédito. Cuenta con una inversión mediante un pagare bancario no negociable por $\$ 110,000.00$ que le genepó intereses al vencimiento de $\$ 3,000.00$ devengados al cie del periodo. Dichos intereses son capitalizables al vencimiento.

Supermercados al Menudeo, S.A.

Estado de Situación Financiera al 31 de agosto de 20.

\begin{tabular}{|c|c|}
\hline $\begin{array}{l}\text { Activo } \\
\text { Circulante }\end{array}$ & $\begin{array}{l}\text { Pasivo } \\
\text { Corto plazo }\end{array}$ \\
\hline Efectivo y Equivalen & Sobregiro de Efectivo \\
\hline$\$ 330,020.00$ & $\$ 3,520.00$ \\
\hline
\end{tabular}

Hermosillo, Sonora a 31 de diciembre de 20...

Notas:

1.) El rubro de efectivo se integra por la cuenta de bancos por $\$ 217,020.00$

2.) El rubro de Equivalente de Efectivo, está integrado por Inversiones Disponibles a la vista mediante un pagaré bancario capitalizable por $\$ 110,000.00$

3.) Existe efectivo restringido en cuenta de Bancos por $\$ 20,000.00$ por concepto de garantizar cumplimiento de cláusulas contractuales con vencimientos mensuales.

as normas de presentación indican que el efectivo debe preentarse como el primer renglón del activo a corto plazo bajo el rubro de Efectivo y equivalente de efectivo.

En notas a los estados financieros debe describirse como se integra este rubro, mencionar el tipo de cambio u unidad de conve

sión, a valor razonable para reflejar el valor de realización
Nota al caso No. 3

El efectivo y equivalente de efectivo se presenta en un solo renglón. Si existe efectivo restringido debe hacer notar este hecho través de notas a los estados financieros, si es a corto plazo la bles debe reflejarse el vararse. En caso de Inversiones disponien este caso es más los intereses ya que son capitalizables a vencimiento. Estos intereses deben reflejarse en el resultado invencimien
tegral.

Los sobregiros bancarios deben presentarse como un pasivo cuando no hay convenio con la institución financiera.
En el supuesto de la situación expuesta en el caso tres, solo que se consideran las variantes marcadas en negrita, quedancontabilidad la siguiente información de sus cuentas de efectivo y equivalentes para la preparación de la información financiera.

En las cuentas de Bancos moneda nacional tiene un saldo de $\$ 213,500.00$ en total, una de estas cuentas muestra un sobre grio de $\$ 3,520.00$. (Existiendo un convenio con la institución fi. de De contrato de servicios, y un contrato firmado compromete a mantener un saldo minimo con retiro condicionado por $\$ 70,000.00$ por un periodo de 18 meses vigentes, hasta que se termine el contrato mencionada para cubrir contingencias y pago de deuda al proveedor). Cuenta con una inversión mediante un pagaré bancario no

\section{Supermercados al Menudeo, S.A.}

Activo

Pasivo

fectivo

Efectivo

No circulante:

fectivo y Equivalente de

fectivo

Restringidos

$70,000.00$

Hermosillo, Sonora a 31 de diciembre de 20.

Notas:

1)El efectivo está integrado por la cuenta de Bancos por $\$ 213,500.00$

2)El equivalente de efectivo por Inversiones disponibles a la vise de efectivo restingidos, es por $\$ 70,000.00$ a un

Notas al caso 4 De acuerdo con la norma vigente, se debe desglosar en notas como se integra el rubro.El efectivo y equivaente de efectivo restringidos, con restricción mayor de un ão debe presentarse por separado fuera del activo a corto plazo, bajo el rubro de Efectivo y Equivalente de efectivo restringidos, manifestando en notas su monto, plazo y tipo de restricción. En caso de sobregiros bancarios bajo convenio con el banco, no se requiere presentarse como un pasivo a corto plazo.Las Inversiones disponibles a la vista se valúan y presentan a su valor de realización que es el mismo monto, ya que los intereses no se capitalizan. Los intereses por $\$ 3,000.00$ deben considerarse dentro del resultado integral. 


\section{Apéndices Técnicos}

Caso 5.

4)El Equivalente de efectivo a largo plazo es por $\$ 100,000.00$ por 2 ños y para el cumplimiento de contrato con proveedores.

Refaccionaria El Tornillo, S.A., manifiesta en su saldo de Ban$\cos \$ 322,500.00$ M.N. (Banamex), existiendo cheques emitidos no entregados por $\$ 11,230.00$,en la cuenta de Bancomer $(\$ 5,400.00)$ no existiendo convenio de compensación. En caja cuenta con 2,300.00 USD valuados al t.c de 12,82, y en HSBC, tiene una caja de seguridad con 4 monedas de metal precioso (oro) valuados originalmente en $5,600.00 \mathrm{c} / \mathrm{u}$ Tiene un pagaré con vencimiento a 60 dias por $\$ 155,000.00$ que le generan intereses por $\$ 4,300.00$ devengados a la fecha de vencimiento no capitalizable. En la cuenta con Banamex existe un fondo restringido por 60 días para garantizar la adquisición de moneda extranjera a t.c. preferente por $\$ 50,000$.00 y otro fondo por $\$ 100,000.00$ por 2 años para garantizar el cumplimiento de contrato con sus proveedores.

El tipo de cambio vigente a la fecha del informe financiero es de $13.10 \times 1$ y la cotización del centenario es de 9,730.00 la pieza.

La situación que se refleja en el balance general sería la si-

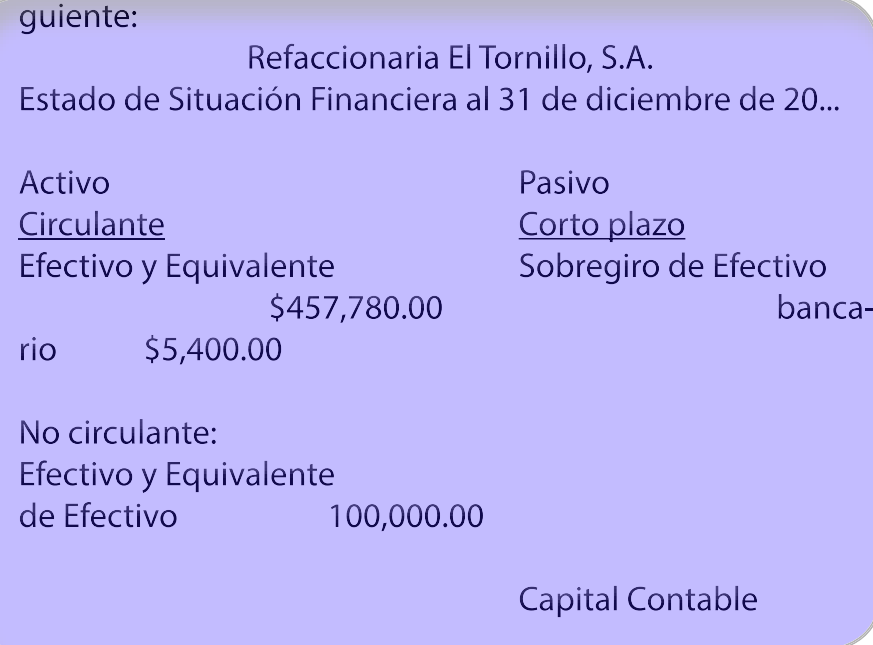

Hermosillo, Sonora a 31 de diciembre de 20...

Notas:

1.El rubro de efectivo está integrado por la cuenta de Bancos con saldo de $\$ 333,730.00$

2.El rubro de Efectivo y equivalente de Efectivo se integra por:

a. Moneda Extranjera por $\$ 30,130.00$ equivalente a 2300

b. Metales preciosos amonedados $38,920.00$ equivalente monedas a $\$ 9730.0 \mathrm{c} / \mathrm{u}$

c. Inversiones disponibles a la vista por $\$ 155,000.00$ con vencimiento a 60 días

3.El efectivo restringido es por $\$ 50,000.00$ por 2 meses para garantizar adquisición de Moneda Extranjera

El Equivalente de efectivo a largo plazo es por $\$ 100,000.00$ por 2 años y para el cumplimiento de contrato con proveedores.
El valor de realización de las inversiones disponibles a la vista es

ta al caso No. 5

De acuerdo con la normatividad se debe mencionar en notas a los Clados financieros las restricciones al efectivo y el tipo de restriccion, Cuando sea mayor de un año debe presentarse en rubro por separado como el caso de los $\$ 100,000.00$, cuando sea menor de un 列 作 pasiva cor deben reintegrarse al saldo de bancos reconociendo el pasivo correspondiente, que en este caso no se refleja en algún pa-

Los sobregiros bancarios en este caso se reconocen como pasivo a corto plazo. La fluctuación cambiaria por $\$ 644.00$ y la diferencia por $\$ 16,520,00$ y los intereses ganados de $\$ 4,300,00$ por la inver sión, se deben considerar en el resultado integral.

\section{Caso 6}

En el caso similar presentado en el caso 5 solo que considerándolas modificaciones que se plantean y muestran en negritaRefaccionaria El Tornillo, S.A., manifiesta en su saldo de Bancos $\$ 322,500.00$ M.N. (Banamex), no existen cheques emitidos sin entregar, en cuenta de Bancomer $(\$ 5,400.00)$ existiendo convenio de compensación. En caja cuenta con 2,300.00 USD valuados al t.c de 12,82, y en HSBC, tiene una caja de seguridad con 4 monedas de metal precioso (oro) valuados originalmente en $5,600.00 \mathrm{c} / \mathrm{u}$., Fondo Fijo de Caja por $\$ 5,000.00$. Tiene un pagare con vencimiento a 60 dias por $\$ 155,000.00$ que le generan intereses por $\$ 4,300.00$ devengados la fecha de vencimiento capitalizable.

En la cuenta con Banamex existe un fondo restringido por 60 días para garantizar la adquisición de moneda extranjera a t.c. preferente por $\$ 50,000.00$ y otro fondo por $\$ 100,000.00$ por 180 días para garantizar el cumplimiento de contrato con sus proveedores.El t.c. vigente a la fecha del informe financiero es de $13.10 \times 1$ y la cotización del centenario es de 9,730.00 la pieza.La situación que se rif Refaccionaria El Tornillo, S.A.
Ren el estado de situaion financiera

$$
\begin{aligned}
& \text { Estado de Situación Financiera al } 31 \text { de julio de 20... } \\
& \text { Pasivo } \\
& \begin{array}{l}
\text { Activo } \\
\text { Circulante }
\end{array} \\
& \begin{array}{l}
\text { Efectivo y Equivalente } \\
\text { Ce Efectivo } \quad \$ 555,850.00
\end{array} \\
& \text { Capital Contable }
\end{aligned}
$$

Hermosillo, Sonora a 31 de julio de 20...
.)El rubro de efectivo está integrado por la cuentas de : Fondo Fijo de Caja por $\$ 5,000.00$

Bancos $322,500.00$

2.)El rubro de Equivalente en efectivo se integra por Moneda Extranjera $\$ 30,130.00$ correspondiente a 2,300 T.C. de $13.10 \times 1$

Metales preciosos amonedados por $38,920.00$ correspondiente a 4 centenarios de oro a $\$ 9,730.00 \mathrm{c} / \mathrm{u}$

3.)Inversiones disponibles a la vista por $\$ 159,300.00$ correspondiente a pagaré bancario a 60 días capitalizable a vencimiento reflejando el valor de realización

4. El efectivo restringido es por $\$ 50,000.00$ a un plazo de 60 días garantía bancaria $100,000.00$ a y un plazo de 180 días como ga-

Nota al caso No. 6

En este planteamiento, el sobregiro bancario no se considera como un pasivo a corto phazo, ya que existe un convenio con Ex

El efectivo restringido todo es a menos de un año, no habiendo necesidad de separarlo, solo se menciona en los notas a los estados financieros. Las inversiones disponibles a la vista, se valúa para efectos de presentación al valor de realización el cual considera los intereses, ya que éstos son capitalizables.

La fluctuación cambiaría por $\$ 644.00$ y la diferencia en cuanto al valor de realización de metales preciosos amonedados por $\$ 16,520.00$, se deben considerar en el resultado integral.
La supervivencia de una empresa reside en gran parte al buen control de sus finanzas. De unas finanzas sanas se logra el éxito en la toma de decisiones. Una empresa que no cuida el rubro de efectivo, difícilmente sobrevivirá. Por ello es recomendable que los administradores de todas las organizaciones le den mayor énfasis al cuidado del dinero, apegándose a la normatividad contable existente. El dinero hace la diferencia entre una empresa en quiebra con una empresa de éxito.

\section{Referencias}

CINIF (2014) NIF C1. Normas de Información Financiera. Consejo Mexicano de Normas de Información Financiera: Instituto

Javier (2014) Principios de Contabilidad. Mc Graw Hill. México 